\title{
Calibration Procedures for Imaging Spectrometers: Improving Data Quality from Satellite Missions to UAV Campaigns
}

\author{
Johannes F. S. Brachmann ${ }^{\mathrm{a}}$, Andreas Baumgartner ${ }^{\mathrm{a}}$, and Karim Lenhard ${ }^{\mathrm{a}}$

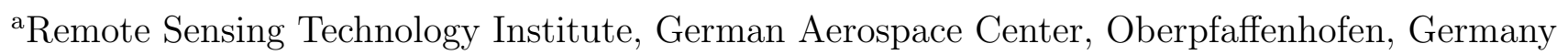

\begin{abstract}
The Calibration Home Base (CHB) at the Remote Sensing Technology Institute of the German Aerospace Center (DLR-IMF) is an optical laboratory designed for the calibration of imaging spectrometers for the VNIR/SWIR wavelength range. Radiometric, spectral and geometric characterization is realized in the CHB in a precise and highly automated fashion. This allows performing a wide range of time consuming measurements in an efficient way. The implementation of ISO 9001 standards ensures a traceable quality of results. DLR-IMF will support the calibration and characterization campaign of the future German spaceborne hyperspectral imager EnMAP. In the context of this activity, a procedure for the correction of imaging artifacts, such as due to stray light, is currently being developed by DLR-IMF. Goal is the correction of in-band stray light as well as ghost images down to a level of a few digital numbers in the whole wavelength range 420-2450 nm. DLR-IMF owns a Norsk Elektro Optikks HySpex airborne imaging spectrometer system that has been thoroughly characterized. This system will be used to test stray light calibration procedures for EnMAP. Hyperspectral snapshot sensors offer the possibility to simultaneously acquire hyperspectral data in two dimensions. Recently, these rather new spectrometers have arisen much interest in the remote sensing community. Different designs are currently used for local area observation such as by use of small unmanned aerial vehicles (sUAV). In this context the CHB's measurement capabilities are currently extended such that a standard measurement procedure for these new sensors will be implemented.
\end{abstract}

Keywords: Hyperspectral, Imaging Spectrometer, Visible-Near Infrared, Short Wave Infrared, Spaceborne, Airborne, UAV, Sensor Characterization, Sensor Model, Calibration, Radiometry, Stray Light, EnMAP, HySpex, CHB

\section{INTRODUCTION}

In recent years, hyperspectral remote sensing of the earth has gained importance on all scales of observation. Improvements in detector technology can significantly enhance measurement capability and also increase the precision of data products. A necessary prerequisite for the latter is an increased stability of the sensors, which can, however, only in combination with a highly precise calibration result in high accuracy data. A precise laboratory characterization and calibration of sensors as diverse as a spaceborne, several airborne and an instrument for small Unmanned Areal Vehicles is in the focus of the work of our group at the Remote Sensing Technology Institute at the German Aerospace Center (DLR-IMF).

This paper is structured into four separate parts:

The first part shortly introduces our calibration laboratory for airborne hyperspectral sensors. Here, standard measurement procedures and the accuracies, with which they are performed, are described. The second part briefly introduces the HySpex airborne sensor system that our institute owns and operates. Further, a short explanation on the working principle of a pushbroom spectrometer is given. The third, which is the longest and most detailed part of the paper, introduces the concept for stray light correction in the EnMAP spaceborne instrument. The method is based on a sensor model that is developed from measurement data. A concept for the measurement of in-band stray light is explained. The fourth part describes some general considerations on hyperspectral sensors for sUAV. Some first new characterization data of the snapshot sensor, that has recently been purchased by DLR-IMF, is shown.

Further author information: Johannes F. S. Brachmann,

E-mail: Johannes.Brachmann@dlr.de 


\section{THE CALIBRATION HOME BASE}

The Calibration Home Base (CHB) is an optical laboratory developed and operated by the German Aerospace Center (DLR) Oberpfaffenhofen for the characterization of (airborne) hyperspectral sensors and field spectrometers. ${ }^{1}$ Radiometric and spectral characterization of cameras is carried out as well.

The CHB was partly funded by the European Space Agency (ESA) to establish a calibration facility for the airborne imaging spectrometer APEX,,$^{2,3}$ but is used for other optical sensors as well. Norsk Elektro Optikks (NEO) HySpex VNIR-1600 and SWIR-320me sensors, which are owned by DLR's Remote Sensing Technology Institute (DLR-IMF) are regularly calibrated in the facility. ${ }^{4}$ It is the only facility in Europe which allows a precise characterization of the radiometric, geometric and spectral properties of bulky and heavy instruments up to $500 \mathrm{~kg}$ (including mechanical interface) in the wide spectral range $380-2500 \mathrm{~nm}$. All standard measurements in the CHB are routinely carried out and are completely automatized. Furthermore, the CHB is also open to requests for special measurement campaigns, if communicated.

Standard measurements in the CHB include spectral measurements in the wavelength range 380-1000 nm, which are performed to a wavelength uncertainty of $\pm 0.1 \mathrm{~nm}$, while an uncertainty of $\pm 0.2 \mathrm{~nm}$ is reached in the wavelength range $1000-2500 \mathrm{~nm}$. Geometric measurements are performed at increments of $1.7 \mu \mathrm{rad}$ across track and $7.6 \mu \mathrm{rad}$ along track. Radiometric measurements reach an absolute uncertainty of $\pm 3 \%(\mathrm{k}=1)$. Sensor artifacts, such as caused by stray light, will be characterizable and correctable in the near future. For now, the CHB is suitable for the characterization of pushbroom sensors, spectrometers and the spectral calibration of cameras. However, it is planned to extend the CHB's capabilities in the near future such that snapshot hyperspectral imagers can be characterized as well.

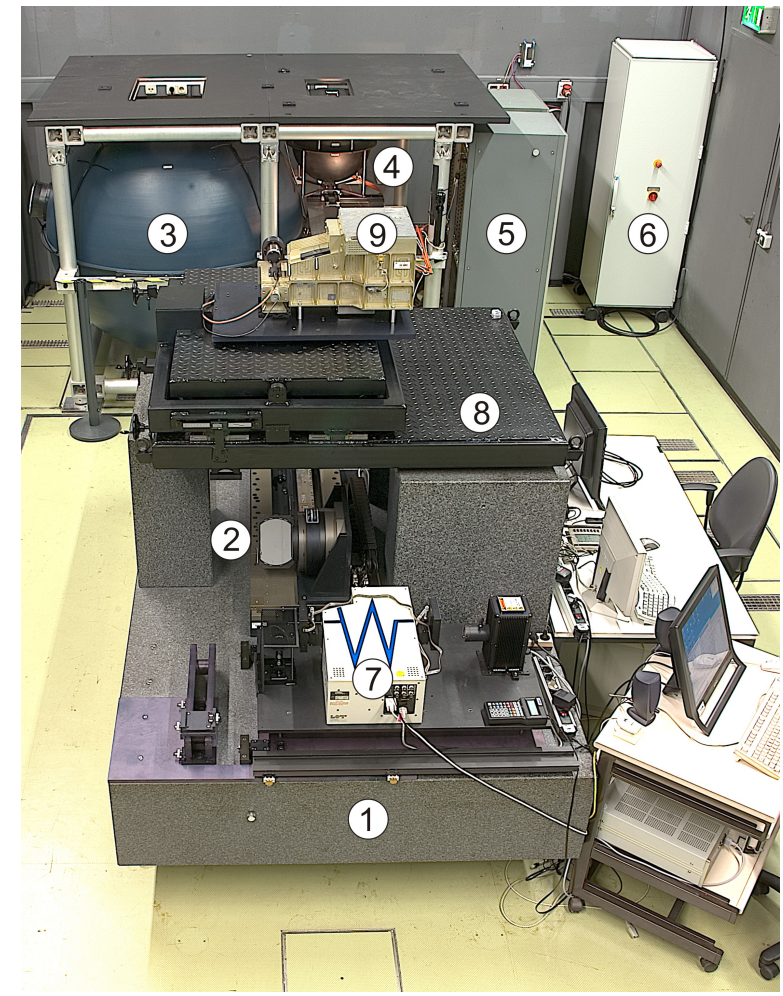

Figure 1. Calibration Home Base (CHB). (1) Calibration bench, (2) Folding mirror, (3) Large integrating sphere, (4) Small integrating sphere, (5) Power supplies of large integrating sphere, (6) Control electronics of folding mirror, (7) Monochromator, (8) CHB adapter, (9) Exemplary sensor. 


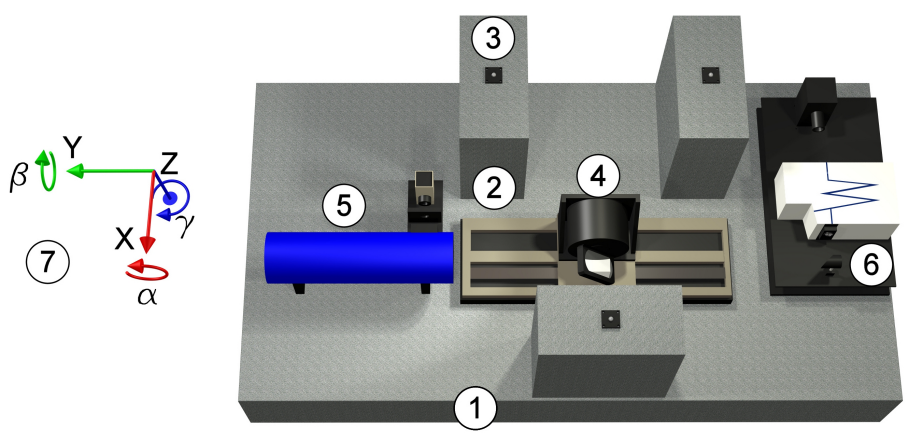

Figure 2. Setup for geometric and spectral measurements. (1) Calibration bench, (2) Pillar carrying sensor, (3) Interface plate, (4) Folding mirror, (5) Components for geometric measurements, (6) Components for spectral measurements, (7) Sensor coordinate system.

\subsection{Laboratory Infrastructure}

Fig. 1 shows the facility with the main measurement equipment. The concept ${ }^{1,5}$ for spectral and geometric measurements with pushbroom imaging spectrometers is illustrated in Fig. 2. A sensor is mounted in a fixed position on top of 3 pillars (2), looking downward onto a mirror (4), which reflects either the beam for geometric (5) or for spectral (6) measurements into the instrument. This 'folding mirror' can be tilted in across-track direction of the sensor in order to set the angle of incidence, and it can be moved in the horizontal direction to meet the entrance aperture. The sensor is attached and aligned on the calibration bench using an adapter. Adapters can be supplied by customers or the general purpose adapter shown in Fig. 1, (8) will be made available. The latter is designed to carry loads of up to $150 \mathrm{~kg}$.

For spectral measurements a monochromator generates a spectrally narrow-band beam of light which is reflected by the folding mirror at well-defined angles into the entrance aperture of the instrument. The collimator setup is used for geometric calibration supplying a nearly parallel beam formed by a lamp-slit-collimator combination.

Two integrating spheres are available for radiometric measurements (see Fig.1). During the measurements, the sensor is mounted on top of the frame either above the small sphere or above the large sphere, depending on the measurement task. The radiance spectrum of both spheres is traceable against German national standard. In order to achieve traceability, the radiance spectrum is constantly monitored against our radiometric standard (RASTA, calibrated at Physikalisch Technische Bundesanstalt) ${ }^{6}$ by the use of a transfer spectrometer (SVC HR1024i). The resulting radiometric spectral $(\mathrm{k}=1)$ uncertainty is $3 \%$ in the spectral range $400-2500 \mathrm{~nm}$.

\section{NEO HYSPEX IMAGING SPECTROMETER SYSTEM}

DLR-IMF owns and operates a commercial airborne imaging spectrometer system produced by Norsk Elektro Optikks A/S (NEO) (see Fig. 3). Consisting of two separate devices (VNIR-1600 and SWIR-320me) the setup covers the spectral range from $400 \mathrm{~nm}$ to $2500 \mathrm{~nm}$.

The HySpex sensors are pushbroom imaging spectrometers, the principle of which is shown in Fig. 4. They are, in effect, line scanners that need to be moved such that successively recorded data can be assembled to two-dimensional data sets. Thus, two orthogonal directions are distinguished: Across-track data are recorded simultaneously in a direction orthogonal to the movement of the instrument. The total viewing angle in this direction is called field of view (FOV). Along-track data is successively recorded line-by-line in direction of the sensor movement. The viewing angle of each detector element is called instantaneous field of view (IFOV). The IFOV is similar to the along-track viewing angle. A slit in an image plane of the optical system constrains these viewing angles. Along the narrow dimension of the slit the light is spectrally unfolded by use of a diffractive or dispersive element. In this manner several wavelength intervals are separately imaged onto a detector array. The two - dimensional array of signals measured by all detector elements that are recorded when imaging the FOV 


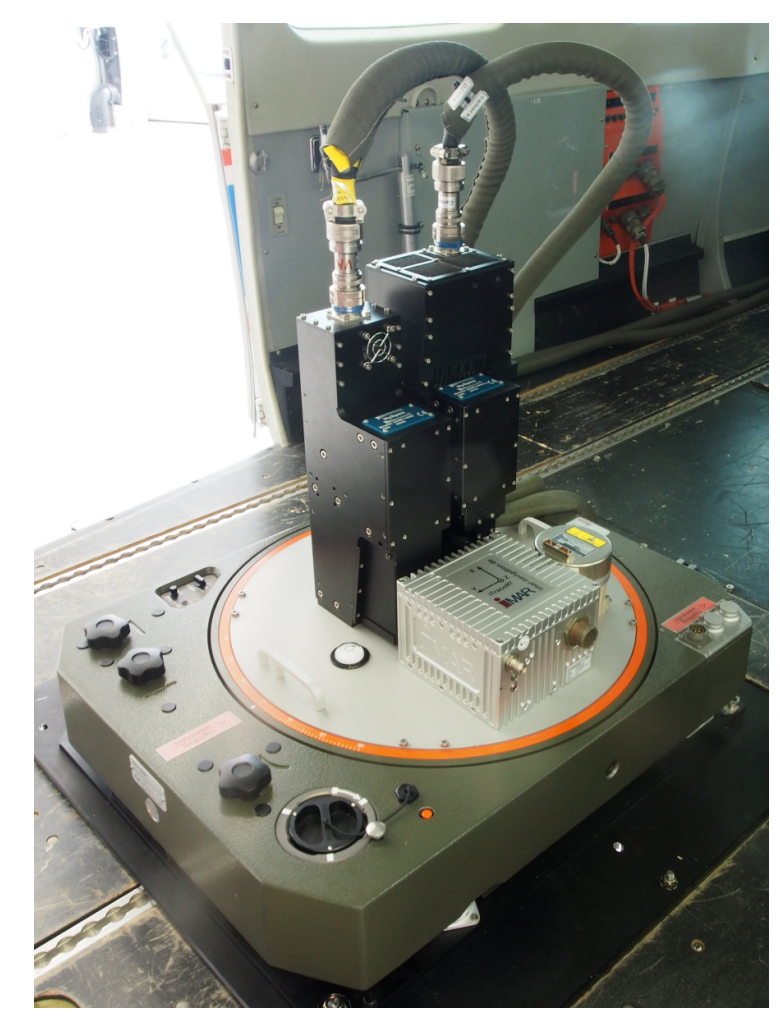

Figure 3. NEO HySpex VNIR-1600 and SWIR-320me mounted in airplane.

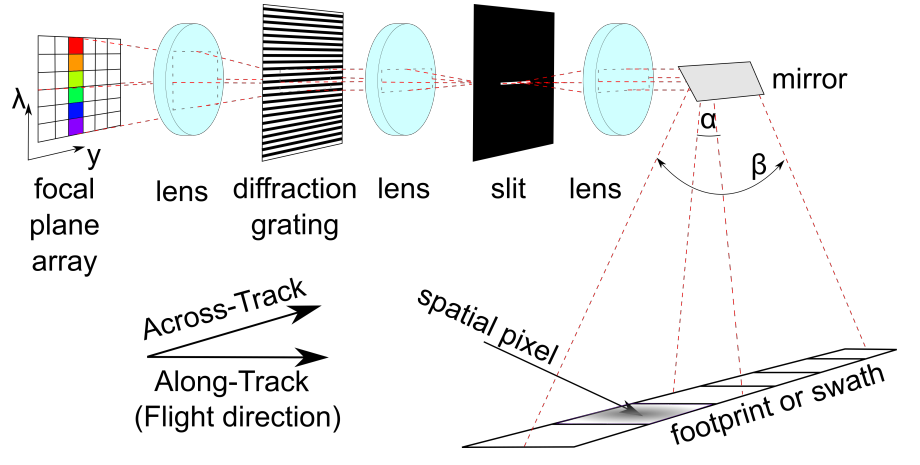

Figure 4. Principle of a pushbroom spectrometer.

is called a frame. One line (y direction in Fig. 4) of the detector array, which receives radiation from a specific wavelength interval, is called channel.

The thorough geometrical and spectral calibration of our HySpex system has been reported previously. ${ }^{7}$ In addition, the stray light in the instrument has been characterized. ${ }^{8}$ The stray light calibration procedure of the spaceborne imaging spectrometer EnMAP will be tested and developed by use of the airborne HySpex imaging spectrometer system. A current state and description of the stray light correction algorithm is described in the following section.

\section{ENMAP CHARACTERIZATION AND CALIBRATION CAMPAIGN}

EnMAP $^{9}$ is a German spaceborne imaging spectrometer mission that is currently in Phase D. Unlike HySpex, EnMAP is a prism spectrometer consisting of two units covering the wavelength range $420-2450 \mathrm{~nm}$. The Visi- 
ble/Near Infrared Spectrometer (VNIR) will operate with 88 channels while the Shortwave Infrared Spectrometer (SWIR) offers 154 channels. The Ground Sampling Distance will be $30 \mathrm{~m}$.

EnMAP's characterization and calibration (C\&C) campaign takes place in a ISO 8 cleanroom environment at OHB System AG and is supported by DLR-IMF. Within this campaign, support for spectral, geometric and radiometric calibration is given. Further, the correction of imaging artifacts, such as due to stray light is the responsibility of DLR-IMF. In the following, the correction methods for the latter, which were developed under contract to OHB System AG, are shortly introduced.

\subsection{Stray Light in the EnMAP Instrument}

Imaging imperfections arise due to different causes. These can be various aberrations of the imaging system, limited aperture and stray light. A measure for the imaging quality of an optical system is the width of its Point Spread Function (PSF). Within this document, the term "stray light" will be used for all imaging artifacts that lead to an increased width of a system's PSF. While not being accurate this is done in order to avoid bulky expressions.

A simulation of an EnMAP VNIR PSF is shown in Fig. 5. A perfect image of a monochromatic radiation source covering a solid angle smaller than the field of view of one detector element would cause signals to be detected only by the respective detector element. However, due to imaging imperfections the optical power is spread out over the detector array.

From the PSF shown in Fig. 5 the stray light levels for various scenes without correction can be estimated. Summing up all the signals except the central peak, a relative stray light level of $0.23 \%$ is found. This corresponds
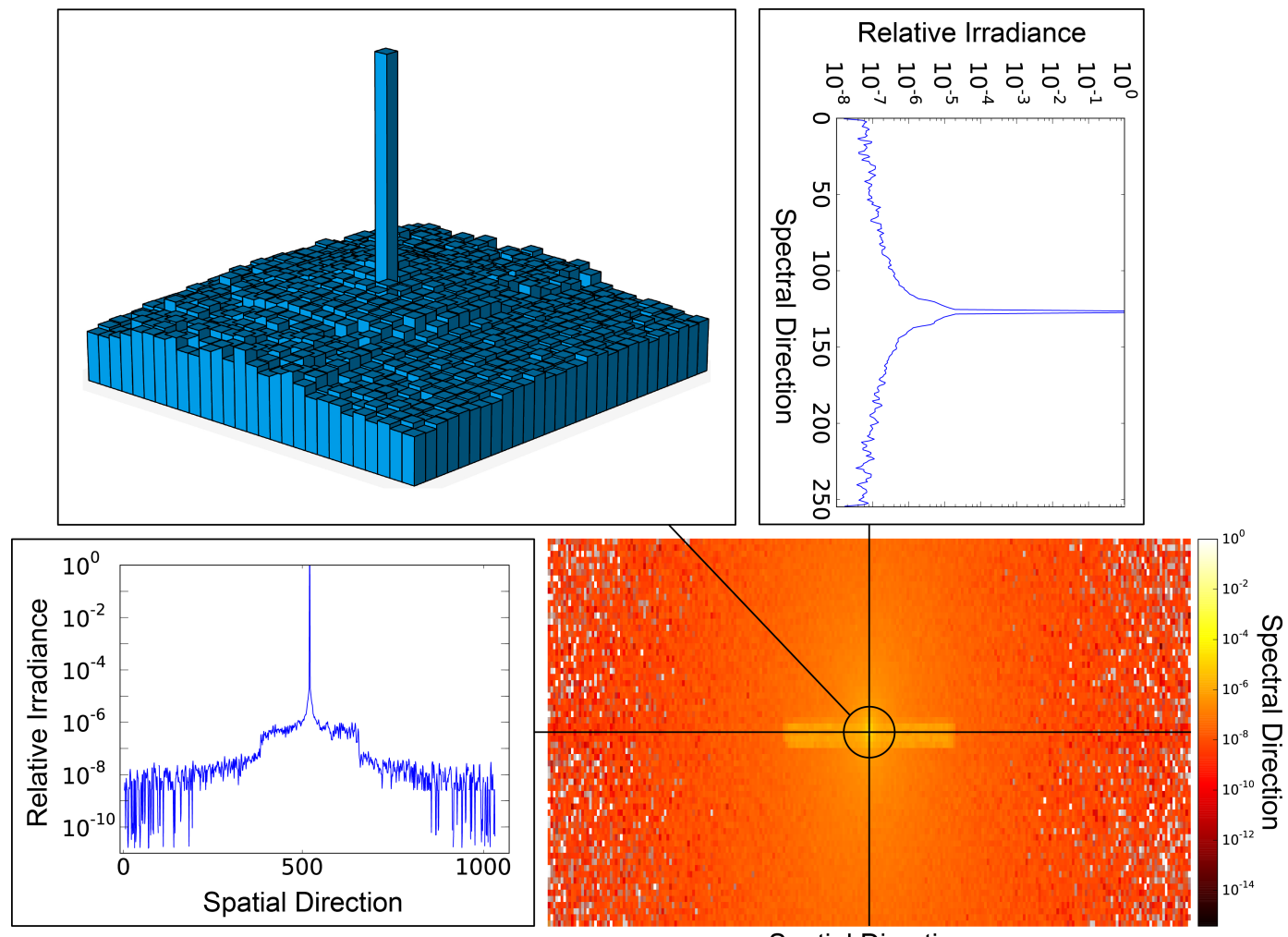

Figure 5. Normalized PSF of VNIR detector at the center of detector array (Pixel 511, Channel 44, corresponding to 659 $\mathrm{nm})$. The size in units of detector elements, shown in spectral direction is larger than the detector. The bar plot shows a central region of the PSF, as well on a logarithmic scale. Here, each bar corresponds to one detector element, with the height of the bar corresponding to the detected signal. The data has been provided by OHB System AG in the context of developing a method for the correction of diffuse stray light in the EnMAP instrument. 
to the relative stray light detected by a single detector element and caused by a homogeneous illumination of all other detector elements.

High optical radiances are recorded during in-flight solar calibration of the instrument. Here, peak values are close to saturation, however not over the whole detector array. This is a consequence of mostly the solar spectrum and the detector quantum efficiency. The average of the detected signal over the whole VNIR detector area during solar calibration is around $50 \%$ of the maximum value corresponding to saturation. The stray light in spatial direction for such a scene can be calculated from the above relative stray light level and assuming a homogeneous irradiance corresponding to $50 \%$ of the maximum value. For such a scene, the stray light detected by each detector element is 7 Digital Numbers (DN). As the spectral radiance of the sun, which has its maximum in the yellow wavelength range, the relative stray light during sun calibration is minimal this wavelength range. In the blue and red wavelength ranges the relative stray light increases, however.

The simulations predict that stray light in the EnMAP instrument is on a very low level and a correction might not be necessary. The goal of the stray light $\mathrm{C} \& \mathrm{C}$ campaign will be to perform measurements in order to confirm or negate these predictions and acquire data for a sensor model for stray light correction if a higher level of the latter is found.

The estimations presented in Secs. 4.2 and 4.3 are based on the data of the VNIR detector shown in Fig. 5. The tails of the PSF of the SWIR detector are on a similar magnitude. At this point, no data of the SWIR detector is presented, as the amount of detail would go beyond the scope of this paper.

\subsection{Stray Light Correction for the EnMAP Instrument}

A simple correction algorithm for a scene in which just one detector element is illuminated is visualized in Fig. 6. The correction would subtract the signals in the outer parts of the PSF and add them to the central peak. In case of a scene containing a more spread out source of radiation, the usual correction algorithm utilizes image deconvolution entailing two Fourier transforms. The correction in the EnMAP instrument will however not be implemented in this manner, but in the way described in the following.

For the purpose of measurement and correction it makes sense to distinguish three different kinds of stray light:

- Out-of-band stray light. Stray light that is caused by light sources outside of the FOV or the spectral range of the instrument.

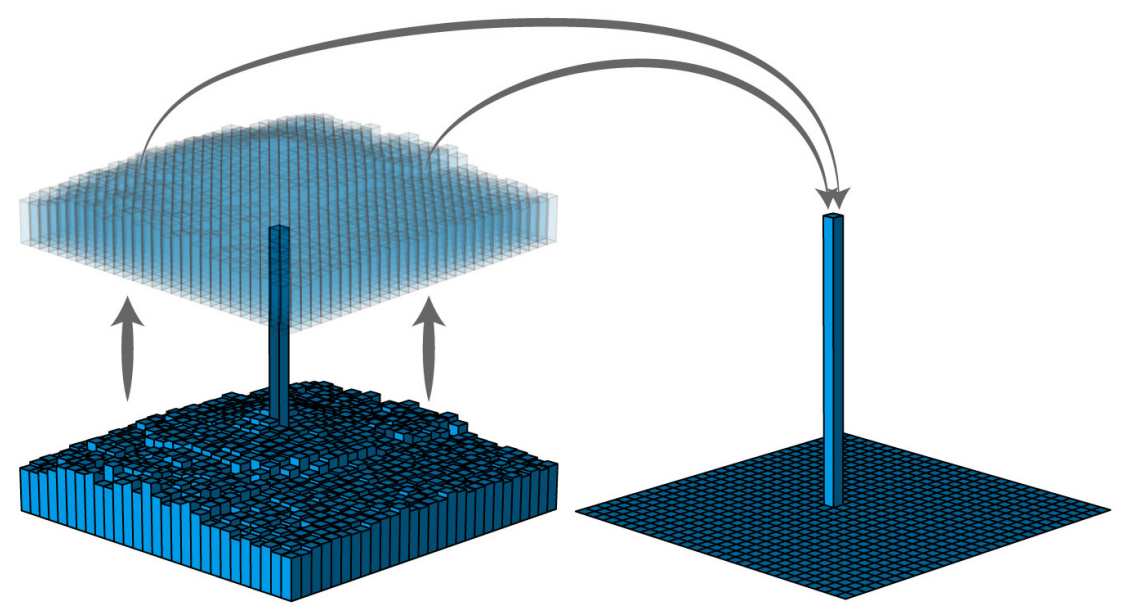

Figure 6. Center part of a scene illuminating one detector element and visualization of a correction in this special case. Each column represents one element of the detector array. The column height is a measure for the signal detected by the respective detector element. 
- Ghost image. Signal originating from a reflection within the spectrometer, which can be directly related to the signal measured by the detector array.

- Diffuse in-band stray light. Signal originating from diffuse reflection, scattering and diffraction. "Inband" means that the stray light is caused by light that enters the instrument within the nominal FOV and spectral range, i.e. that a relationship between the measured signal and its stray light component exists.

The stray light correction will be implemented for each of these components separately in the order given above.

\subsubsection{Diffuse in-band stray light}

In the case of non-imaging spectrometers diffuse in-band stray light can be corrected using the approach proposed in ${ }^{10}$. Our correction algorithm is based on the same method, but extended for the correction of imaging spectrometer data.

At the core of the algorithm is the stray light correction tensor $\mathbf{C}=\mathbf{D}^{-1}$, which is the inverse of the stray light tensor D. D consists of the signals measured at all detector elements when a single detector element is illuminated - for each detector element. $\mathbf{C}$ and $\mathbf{D}$ are normalized tensors of rank 4 . The correction is achieved by a simple tensor multiplication

$$
\mathbf{S}^{\text {corr }}=\mathbf{C} \otimes \mathbf{S}
$$

where $\mathbf{S}$ is the recorded image frame and $\mathbf{S}^{\text {corr }}$ is the corrected frame.

In the case of EnMAP data, the stray light correction will be part of the Level 0 (L0) processor. As such, data needs to be processed in close to real time. However, the correction tensor, if the correction is carried out on the full resolution of the detector array, is too large to be processed on the the given timescale. Thus, a binning concept will be used for correction. This means, the correction is done on a coarser grid of detector elements.

\subsubsection{Ghost images}

Ghost images can induce stronger signals than diffuse stray light and are constrained to certain detector elements. While for diffuse in-band stray light significant detector signals are reached through the addition of the far reaching tails of all PSFs, ghost images originate from reflections occurring in the optical assembly. For a correction of the latter, origin $\mathbf{O}^{k}$ and target $\mathbf{G}^{k}$ detector maps are generated for each occurring ghost image $k$. An origin detector map comprises of detector elements that, when illuminated, detect signals relating to a ghost image detected by other detector elements. Ghost target maps comprise the signal detected on elements where a ghost image is observed. Detector elements in both maps that do not register signals are set zero. Each ghost image is corrected individually and successively using its own maps $\mathbf{O}^{k}$ and $\mathbf{G}^{k}$ which have dimension of the detector array.

For the correction, the intensity $p$ of the ghost image $k$ is determined by multiplying $\mathbf{O}^{k}$ with the signal frame $\mathbf{S}$ component-wise, and forming their average:

$$
p^{k}=\frac{1}{N} \sum_{i, j} O_{i, j}^{k} \cdot S_{i, j}
$$

where $N$ is the number of nonzero elements in $\mathbf{O}^{k}$ and the sum is carried out over all detector elements. The ghost-corrected frame $\mathbf{S}^{\text {corr }}$ is then obtained using the relation

$$
S_{i, j}^{\mathrm{corr}}=S_{i, j}-\sum_{k} p^{k} \cdot G_{i, j}^{k} .
$$




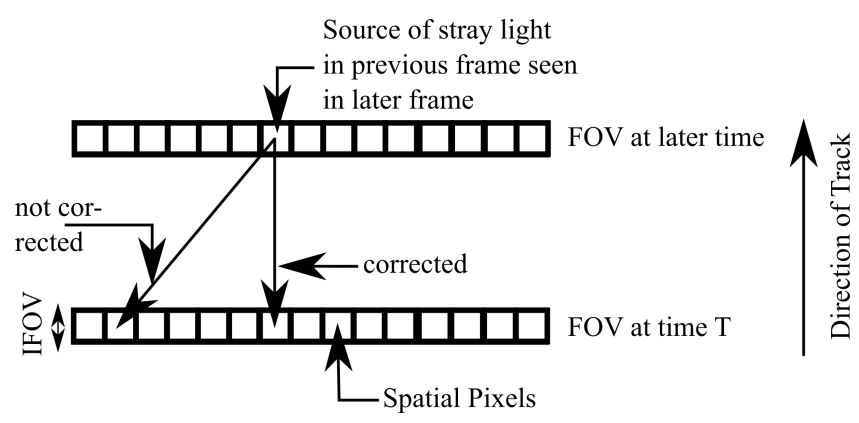

Figure 7. 1 dimensional correction algorithm for spatial along-track out-of-band stray light.

\subsubsection{Out-of band stray light}

It is only in few cases possible to correct out-of band stray light, as the stray light signal usually does not relate to any measured signal. However, a simple approach could correct spatial along track stray light from sources outside the field of view. Such sources could be clouds or reflecting surfaces in a frame recorded in the same flight line.

The one-dimensional correction algorithm is illustrated in Fig. 7. If one assumes an unchanging scene, successively recorded frames can be used for a correction of each other. In this manner, if a bright source is recorded in one or more frames in a data take, its influence on other frames that are outside the instantaneous field of view can be quantified.

\subsection{Measurement of Diffuse In-Band Stray Light in the EnMAP Instrument}

As described in Sec. 4.2.1 the measurement values used in the correction algorithm are the elements of the stray light tensor D. In order to measure the elements of $\mathbf{D}$, the response of all detector elements, when a certain

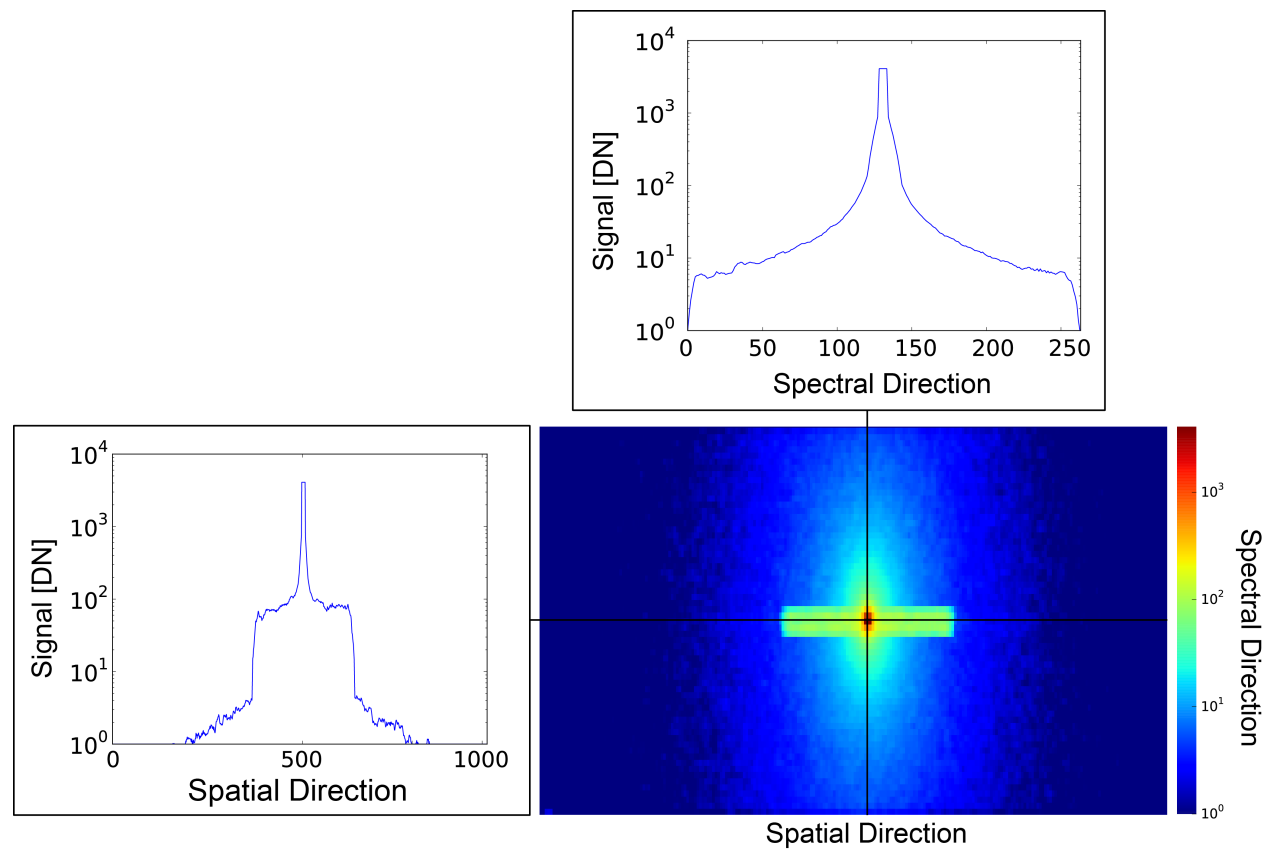

Figure 8. Prediction of signal levels of the saturated frame during a stray light bracketing measurement of the VNIR detector in DN (12 bit sensor). Within the illuminated detector area an irradiance corresponding to a factor of 2000 above saturation is assumed. Further parameters are: detector integration time of $4 \mathrm{~ms}$, illuminated area $9 \times 6$ (spatial pixels $\times$ spectral channels). 
detector element or area on the detector is illuminated, needs to be recorded. In order to measure stray light signals of detector elements located at large distance from the illuminated area (outer area of the PSF) more than seven orders of magnitude need to be "lifted up" into the detectable range (see Fig. 5). To achieve this, a bracketing technique is employed, for which two or more frames with different at-detector irradiances are recorded and later combined. Low irradiances allow the measurement of the center parts of the PSF, but not the signals in the outer parts. High irradiances will saturate the detector at peak levels, but allow the measurement of the outer parts of the PSF.

The light power above saturation that needs to be reached in order to make the tails of the PSF visible depend on the size of the illuminated spot on the detector. Illuminating a larger spot will generate more stray light, which can help keeping the optical power below the damage threshold for detectors, while still allowing the measurement of stray light with sufficient dynamics. It is foreseen to measure stray light in the EnMAP instrument at a level of around $1-3 \%$ of the saturated signal. An area of $9 \times 6$ (spatial pixels $\times$ spectral channels) will be illuminated. Fig. 8 shows a prediction for the signal strengths in the saturated frame (high irradiance bracketing frame) in digital numbers (DN). This prediction is based on the PSF shown in Fig. 5 and for the nominal integration time of $4 \mathrm{~ms}$. It is to be noted that the integration time can be chosen longer. Thus, the estimation of signals presented in Fig. 8 corresponds to settings in which the lowest levels of stray light are measured. However, for the SWIR detector, the amount by which the integration time can be increased, is limited to a factor of around 5. Thus, the estimates that are shown are on the order of the predicted signal levels of the measurement for the SWIR detector.

\section{SMALL SCALE HYPERSPECTRAL REMOTE SENSING BY USE OF UNMANNED AREAL VEHICLES}

Currently, there is a lot of interest in the rapidly growing field of hyperspectral remote sensing by use of small Unmanned Areal Vehicles (sUAV). Indeed, using hyperspectral remote sensing on sUAV can bridge a gap between field spectrometers and airborne spectrometers. Low cost campaigns and flexibility of operation make several applications feasible for the first time. Up to now agriculture/vegetation monitoring and the monitoring of industrial facilities have been in the focus of businesses providing operative services. Many companies, that are established in the field of airborne spectroscopy, enter the field with products that resemble down-scaled pushbroom sensors similar to the ones used in airplanes. These companies are joined by startups that are offering products based on new working principles.

When deciding which of the many options of imagers to acquire the legislation of the target operating location of the UAV is important to consider. In Germany a general take off permit can be issued for sUAV with a total take off-mass below $5 \mathrm{~kg}$. For the operation of heavier UAV a permit needs to be acquired for every flight campaign.

Naturally, at least equally important to legislative considerations are the applications for which one wants to use the imager. There is a wide variety of lightweight imagers available, some of which possess unique properties. A small selection of imagers we found particularly interesting is shown in Tab. 1. Cubert and Rikola instruments are currently the only snapshot imagers with Signal to Noise Ratios (SNR) that are comparable to the available pushbroom instruments. These allow the hyperspectral recording of dynamic 2 dimensional scenes and even

Table 1. Overview over several different lightweight instruments for sUAV.

\begin{tabular}{llcl}
\hline Sensor & Principle & Wavelength Range $[\mathrm{nm}]$ & Channels $\times$ Pixels \\
\hline Cubert UHD-185 & Snapshot & $450-950$ & $125 \times 50 \times 50$ \\
Rikola HSC & Snapshot & $500-900$ & 50 narrow, freely selectable $\times$ \\
& & & $648 \times 1010 \times$ \\
Headwall Micro E_Series & Pushbroom & $400-1000$ & $370 \times 1600$ \\
Headwall Micro X_Series & Pushbroom & $550-1650$ & $96 \times 320$ \\
Resonon Pika NUV & Pushbroom & $350-800$ & $184 \times 1600$ \\
Headwall SWIR M_Series & Pushbroom & $900-2500$ & $267 \times 640$
\end{tabular}




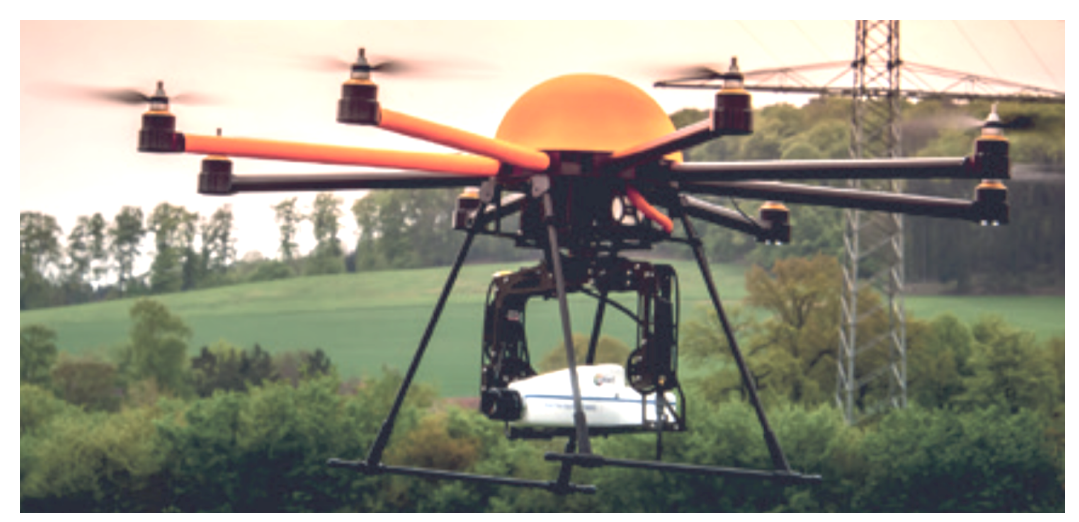

Figure 9. Snapshot hyperspectral camera Cubert UHD-185 mounted on sUAV. The image is supplied by Cubert GmbH.

hyperspectral video. The Headwall devices stand out through very wide wavelength ranges combined with high spectral and spatial resolution. The Resonon instrument is especially interesting for remote sensing of water areas, due to its wavelength range that reaches to short wavelengths - even into the ultraviolet.

Within DLR's Earth Observation Center several groups are interested in testing the suitability of one or several of these devices for a wide variety of applications. The latter include remote sensing of inland water areas, vegetation \& soil, monitoring traffic and building conditions, applications in crisis information as well as using the device for calibration and validation measurements of satellite and airplane hyperspectral data.

DLR-IMF has very recently purchased a Cubert UHD-185 hyperspectral imager* as well as an sUAV with a total take off mass (instrument $+\mathrm{sUAV}$ ) of $5 \mathrm{~kg}$. The instrument, together with an sUAV, which is however of a different make than the one purchased, is shown in Fig. 9. As the sensor has only recently arrived, it is currently tested, calibrated and mounted on a gimbal for the sUAV.

The Cubert instrument is a prism based design. ${ }^{11}$ If not corrected, dispersive unfolding leads to channel widths that vary widely with wavelength. This has been confirmed in the measurement shown in Fig. 10. For the measurement, the monochromator setup shown in Fig. 1, has been employed. Light with the specified arbitrarily

${ }^{*}$ http://cubert-gmbh.de/

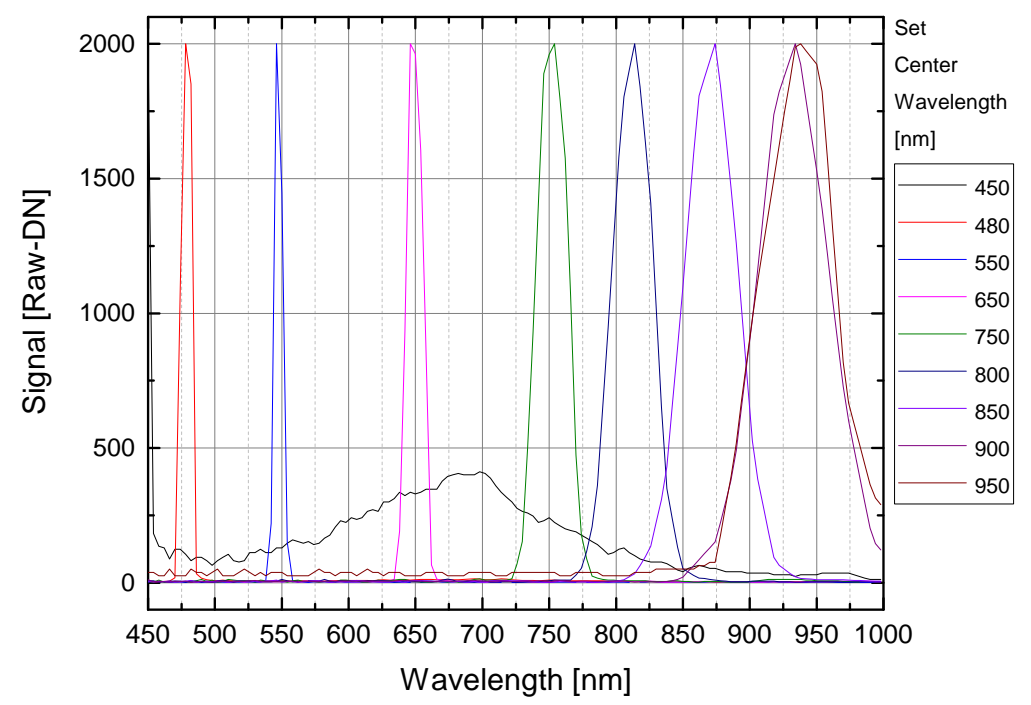

Figure 10. Response of the Cubert UHD-185 imager to spectrally narrowband illumination. The measurement was done with the CHB monochromator with center wavelengths accurate to $0.1 \mathrm{~nm}$ and FWHM spectral width of $1 \mathrm{~nm}$. 
chosen wavelengths and at an arbitrarily chosen hyperspectral pixel was analyzed by the instrument. It is found that while the specification for the channel width at $550 \mathrm{~nm}(\sim 8 \mathrm{~nm}$ FWHM $)$ is satisfied, a far wider spectral response is observed in the longer wavelength range. Further, the central wavelengths show deviations. The latter might be corrected by a recalibration. The black function (corresponding to $450 \mathrm{~nm}$ ) shows that this wavelength is slightly out-of-band. The signal shown is thus the out-of-band stray light of the system at the chosen pixel coordinate.

It needs to be pointed out that the Cubert instrument is a unique and new design. DLR-IMF and Cubert $\mathrm{GmbH}$ plan to further characterize the instrument in the CHB. In this manner the new device will be developed further and its performance optimized.

\section{CONCLUSION}

High precision remote sensing data products require a thorough characterization of a wide variety of sensor parameters. Moreover, an in-flight monitoring of spaceborne and airborne sensors as well as a recalibration schedule are indispensable for generating reliable high level data products. In many cases only the above allows replicable statements to be made in a field in which it is very difficult to acquire precise data.

This paper shortly introduces characterization measurements for imaging spectrometers that are routinely carried out in our optical laboratory. These include spectral, geometric, radiometric, linearity and polarimetric characterization. Most of the acquired data can be used for a correction of the respective sensor artifacts. Other measurements, such as stray light characterization as well as the extension of measurement capabilities for new types of sensors are in the focus of current activities.

Depending on the application, different sensor artifacts influence the accuracy of the data more. It is thus important to consider which of the many possible characterizations should be completed. In addition to radiometry, standard measurements that are almost always carried out are spectral and geometric corrections. Stray light can produce large radiometric errors, but is more difficult to measure, especially in instruments in the SWIR wavelength range.

It is to be noted that the succession of the correction of artifacts is of great importance. A well thought through order of L0 processors need to be implemented for correction, as the application of some calibration steps requires other steps to be carried out beforehand. Further, it needs to be ensured that different measurements, which might detect similar effects do not correct the same effect twice. An example for this is the successive correction of the different types of stray light, which is described in this paper. For this, a correction concept is introduced.

The CHB and the airborne HySpex sensors are made available to the public through the user service Optical Airborne Remote Sensing and Calibration Home Base (OpAiRS) ${ }^{\dagger}$.

\section{ACKNOWLEDGMENTS}

The authors would like thank the remaining CHB team Thomas Schwarzmaier, Peter Gege and Claas Köhler for their contributions to the laboratory and numerous discussions.

The development of a stray light correction method for the EnMAP instrument was completed for the EnMAP prime contractor OHB System AG who is under contract to DLR with funding from the German Federal Ministry for Economic Affairs and Energy (Bundesministerium für Wirtschaft und Energie) under reference number 50 EP 0801 .

\footnotetext{
${ }^{\dagger}$ www.dlr.de/opairs
} 


\section{REFERENCES}

[1] Brachmann, J. F. S., Andreas, B., and Gege, P., "The calibration home base for imaging spectrometers," Journal of large-scale research facilities 2, A82 (2016).

[2] Itten, K. I., 'Endice, F. D., Hueni, A., Kneubühler, M., Schläpfer, D., Odermatt, D., Seidel, F., Huber, S., Schopfer, J., Kellenberger, T., Bühler, Y., D'Odorico, P., Nieke, J., Alberti, E., and Meuleman, K., "APEX - the hyperspectral ESA airborne prism experiment," Sensors 8, 6235 - 6259 (Oktober 2008).

[3] Schaepman, M. E., Jehle, M., Hueni, A., D’Odorico, P., Damm, A., Weyermann, J., Schneider, F. D., Laurent, V., Popp, C., Seidel, F. C., Lenhard, K., Gege, P., Küchler, C., Brazile, J., Kohler, P., Vos, L. D., Meuleman, K., Meynart, R., Schläpfer, D., Kneubühler, M., and Itten, K. I., "Advanced radiometry measurements and Earth science applications with the Airborne Prism Experiment (APEX)," Remote Sensing of Environment 158, 207 - 219 (2015).

[4] Lenhard, K., Baumgartner, A., and Schwarzmaier, T., "Independent laboratory characterization of NEO HySpex imaging spectrometers vnir-1600 and swir-320m-e," Geoscience and Remote Sensing, IEEE Transactions on 53(4), 1828-1841 (2015).

[5] Sümnich, K. H., "Vorrichtung zur Kalibrierung eines optischen Instruments mit einem fotoelektrischen Detektor.," German Patent Application Publication (2003). DE 10218947 A 1.

[6] Schwarzmaier, T., Baumgartner, A., Gege, P., Köhler, C., and Lenhard, K., "The radiance standard RASTA of DLR's calibration facility for airborne imaging spectrometers," Proc. SPIE 8533, 85331U-85331U-6 (2012).

[7] Baumgartner, A., Gege, P., Köhler, C., Lenhard, K., and Schwarzmaier, T., "Characterisation Methods for the Hyperspectral Sensor HySpex at DLR's Calibration Home Base," (2012).

[8] Lenhard, K., Baumgartner, A., Gege, P., Nevas, S., Nowy, S., and Sperling, A., "Impact of improved calibration of a NEO HySpex vnir-1600 sensor on remote sensing of water depth," IEEE Transactions on Geoscience and Remote Sensing 53(11), 6085-6098 (2015).

[9] Guanter, L., Kaufmann, H., Segl, K., Förster, S., Rogass, C., Chabrillat, S., Küster, T., Hollstein, A., Rossner, G., Chlebek, C., Straif, C., Fischer, S., Schrader, S., Storch, T., Heiden, U., Müller, A., Bachmann, M., Mühle, H., Müller, R., Habermeyer, M., Ohndorf, A., Hill, J., Buddenbaum, H., Hostert, P., van der Linden, S., Leitão, P. J., Rabe, A., Dörffer, R., Krasemann, H., Xi, H., Mauser, W., Hank, T., Locherer, M., Rast, M., Stänz, K., and Sang, B., "The EnMAP spaceborne imaging spectroscopy mission for earth observation," Remote Sensing 7(7), 8830-8857 (2015).

[10] Zong, Y., Brown, S. W., Johnson, B. C., Lykke, K. R., and Ohno, Y., "Simple spectral stray light correction method for array spectroradiometers," Appl. Opt. 45, 1111-1119 (Feb 2006).

[11] Jung, A., Michels, R., and Graser, R., "Räumlich und spektral auflösende Hyperspektralkamera und Verfahren," European Patent Application Publication (2015). EP 2944930 A3. 